\title{
Cerebral Myxomatous Aneurysm Treated by M2-M2 Bypass: A Case Report
}

\author{
Hee Jun Yoo, Jaewoo Chung, Jae Sung Ahn \\ Department of Neurosurgery, Asan Medical Center, University of Ulsan College of Medicine, Seoul, Republic of Korea
}

Corresponding author:

Jae Sung Ahn

Department of Neurosurgery,

Asan Medical Center, University of Ulsan College of Medicine,

88, Olympic-ro 43-gil,

Songpa-gu, Seoul 05505,

Republic of Korea

Tel: +82-2-3010-3550

Fax: +82-2-476-6738

E-mail: jsahn@amc.seoul.kr

Received: September 9, 2018

Revised: September 13, 2018

Accepted: September 17, 2018

\begin{abstract}
Intracranial aneurysms associated with atrial myxoma have been reported and termed "myxomatous aneurysms." We present a case of cerebral myxomatous aneurysms treated by M2-M2 bypass surgery. A 20-year-old woman was admitted for evaluation and management of multiple cerebral aneurysms found incidentally. She had a history of right middle cerebral artery (MCA) territory infarction with MCA total occlusion. At that time, cardiac myxoma was incidentally found and surgically removed. In cerebral angiography, multiple cerebral aneurysms were identified. Among them, a right MCA fusiform aneurysm was the largest, with a diameter of $19.5 \mathrm{~mm}$; notably, this developed at the site of previous MCA occlusion. Through surgical exploration, an MCA bifurcation aneurysm was observed, with a permanently occluded inferior trunk toward the temporal lobe. M2-M2 end-to-end anastomosis with resection of aneurysm was successfully performed, and biopsy showed dilation and thickening of the aneurysmal wall without any evidence of tumor invasion. The outcome of this case shows that surgical resection with bypass surgery is a useful option for the treatment of cerebral myxomatous aneurysms.
\end{abstract}

Key Words: Cardiac surgical procedures; Heart atria; Intracranial aneurysm; Myxoma

\section{INTRODUCTION}

Intracranial aneurysms associated with atrial myxoma have been reported and termed "myxomatous aneurysms". Because of their infrequent incidence, treatment strategy for multiple myxomatous aneurysms is not well-established. Here, we present a case of cerebral myxomatous aneurysms in our hospital treated by M2-M2 bypass surgery.

\section{CASE REPORT}

A 20-year-old woman was admitted to our institute for evaluation and management of multiple cerebral aneurysms that were found incidentally. She had a history of sudden onset of left side motor weakness 4 years prior; magnetic resonance (MR) angiogram had confirmed right middle cerebral artery (MCA) total occlusion, with MCA territory infarction. Intravenous tissue-type plasminogen activator and thrombolysis was administered, and the MCA was recanalized. Echocardiography revealed a cardiac mass that was $3 \mathrm{~cm}$ in maximum diameter; the mass was removed and confirmed as cardiac myxoma by pathology evaluation. She recovered from motor weakness, but lost vision in her right eye due to this event.

By using cerebral angiography, multiple cerebral aneurysms were identified (Fig. 1). Among them, a right MCA fusiform aneurysm was the largest, with a diameter of $19.5 \mathrm{~mm}$; it had developed at the site of the previous MCA occlusion. Considering the risk of rupture, surgery was planned with preparation for superficial temporal artery-MCA bypass. During surgical exploration, the MCA aneurysm, which had been suspected as a fusiform aneurysm, was identified as an MCA bifurcation aneurysm with permanently occluded inferior trunk toward the temporal lobe. The aneurysm exhibited a rubber-like feature; notably, a feasible resolution was M2-M2 end-to-end anastomosis with resection of aneurysm (Fig. 2). Anastomosis was successfully performed, and biopsy showed dilation and thickening of the aneurysmal wall without any evidence of tumor invasion.

\section{DISCUSSION}

Cardiac myxoma is the most common primary heart neoplasm, constituting 50\% of all cardiac tumors; its incidence is approximately 0.5 cases per $1,000,000$ population per year ${ }^{1)}$. There are two main types. Most myxomas are smooth, firm masses that can be calcified or ossified. A small proportion of myxomas are soft, gelatinous tumors with irregular fronds; these often embolize. Although they are benign tumors with a classical risk of metastasis, such embolization can cause is- 
chemic events or aneurysms ${ }^{4)}$.

Neurologic complications associated with cardiac myxoma are observed in up to $35 \%$ of patients ${ }^{6}$. In the literature, symp- toms of stroke or peripheral ischemia due to embolization are reported in approximately $22 \%$ of myxoma cases. The incidence of embolization is not related to the size of the tumor,
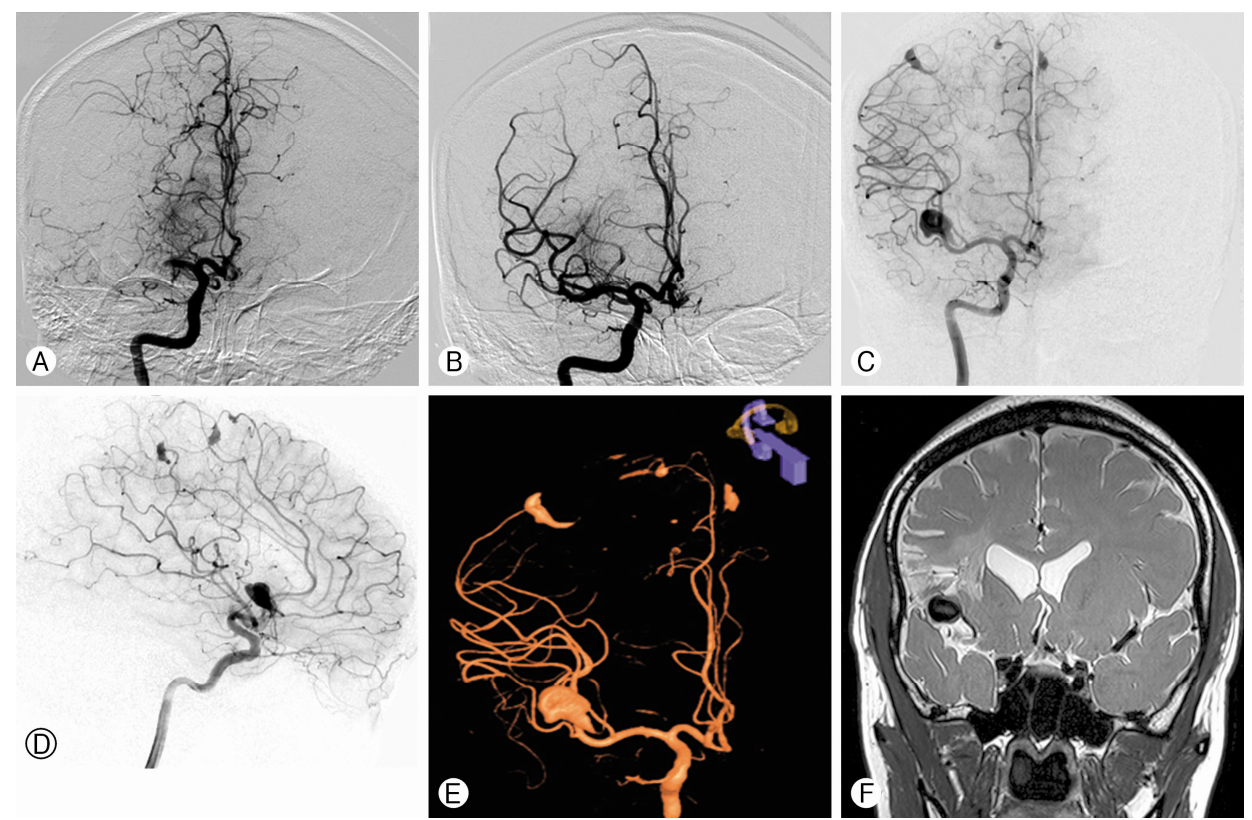

Fig. 1. (A) Right internal carotid artery digital subtraction angiogra- phy showing right middle cerebral artery (M1 segment) occlusion. (B) Reperfusion via right middle cerebral artery after intravenous tissuetype plasminogen activator injection. (C, D) Right internal carotid ar- tery digital subtraction angiography anteroposterior and lateral views showing multiple aneurysms. (E) Right middle cerebral artery bifurcation aneurysm was $19.5 \mathrm{~mm}$ in maximum diameter on 3-dimensional reconstruction image. (F) T2weighted magnetic resonance image showing large aneurysm at the right middle cerebral artery.
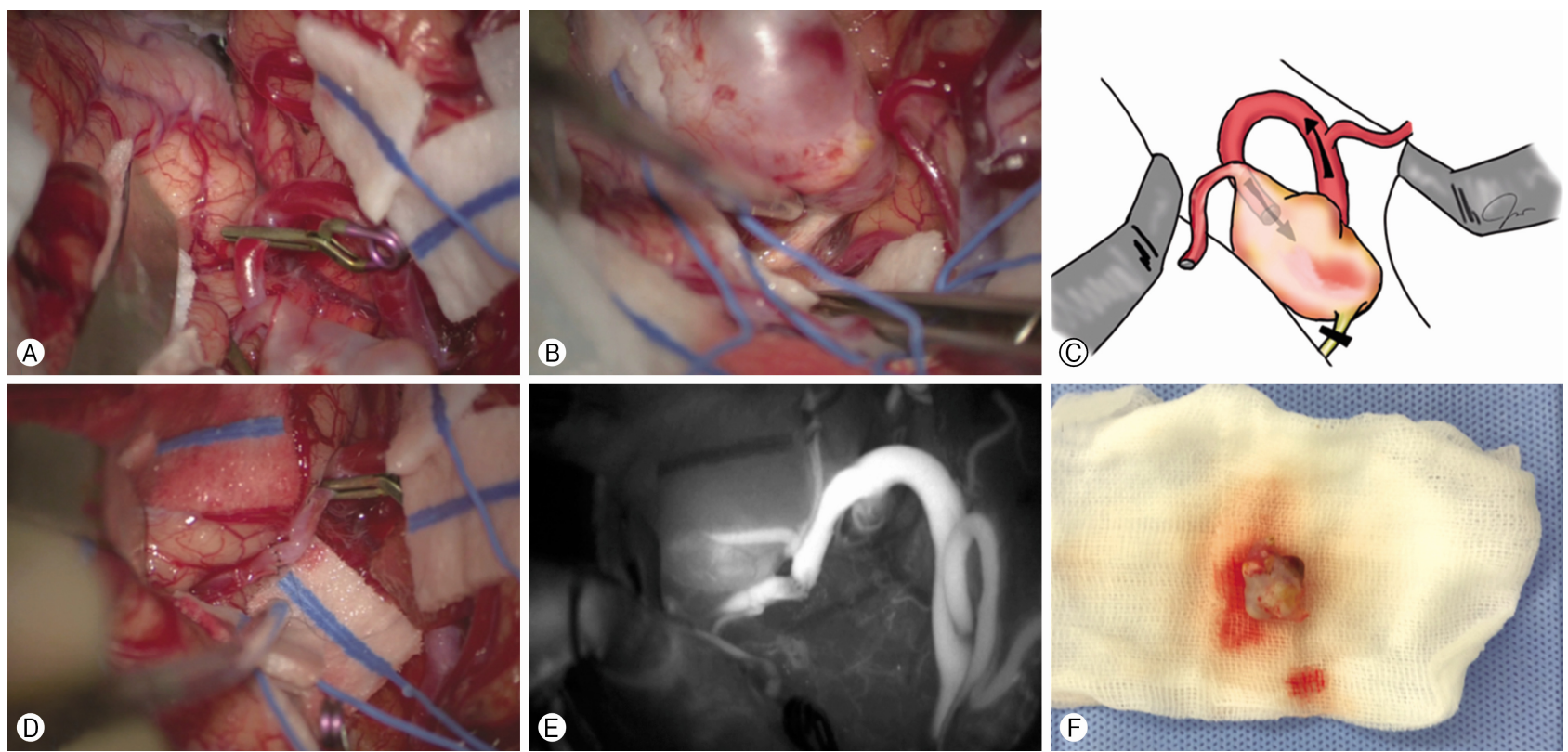

Fig. 2. (A-C) Bifurcated middle cerebral artery aneurysm with permanently occluded inferior trunk toward temporal lobe was observed during surgical exploration. (D, E) M2-M2 end-to-end anastomosis with resection of aneurysm was successfully performed. (F) Biopsy showed dilation and thickening of the aneurysmal wall. 
but is instead related to tumor mobility and friability ${ }^{1,10)}$.

Myxomatous aneurysms, intracranial aneurysms associated with cardiac myxoma, are less common; their true incidence is unknown, but reports of such aneurysms are increasing with improvements in cardiac and neurovascular imaging that facilitate early detection of disease ${ }^{8)}$. The mechanism for the formation of intracranial aneurysms in conjunction with cardiac myxomas is not well-established, but some hypotheses have been proposed: (1) Myxomatous emboli can block the vasa vasorum, destroying the normal architecture of the vessel, similar to mechanism of mycotic aneurysms; the vessel wall becomes vulnerable to ischemic injury and subsequent dilatation, which lead to aneurysm formation, ${ }^{2,8)}$; (2) Myxomatous emboli produce vascular occlusion, causing scarring and pseudoaneurysm formation; (3) Tumor cells can proliferate and penetrate the wall; either mechanism can lead to scarring of the vessel wall and pseudoaneurysm formation ${ }^{11,13)}$. The angiographic characteristics of myxomatous aneurysms are multiplicity, fusiform appearance, and distal location. These features are similar to those of immunodeficiency-associated aneurysms and septic emboli-causing aneurysms ${ }^{5,7,16)}$.

Treatment options for myxomatous aneurysms are conservative care, endovascular methods, or surgery. These aneurysms may remain stable for many years; additionally, spontaneous regression of intracranial myxomatous aneurysms has been frequently reported ${ }^{9,12,14)}$. Therefore, without a specific risk factor, conservative treatment with serial MR imaging or angiography follow-up can be a reasonable strategy, especially when the risk of surgical treatment is high ${ }^{15}$. Cases of ruptured aneurysm are generally considered urgent surgical candidates. Because of the fusiform shape of most myxomatous aneurysms, it is difficult to perform conventional clipping of the aneurysm while maintaining the flow of the parent artery; bypass surgery is a reasonable option when sacrifice of the feeding artery may be required. In this case, M2-M2 end-to-end anastomosis was performed with complete resection of the myxomatous aneurysm. However, bypass surgery is technically challenging, compared with other options, and is limited because it is difficult to apply in a variety of locations. Additional case studies are needed regarding the location of myxomatous aneurysms and successful surgical approaches. Endovascular treatment is a reasonable treatment option. Because of the fusiform shape, coil embolization with stenting could be necessary ${ }^{7)}$. There remains much debate regarding the possibility that chemotherapeutics can stabilize myxomatous aneurysms when surgery or endovascular procedure is not possible ${ }^{3)}$.

In conclusion, our case shows that surgical resection with bypass surgery is a useful option for the treatment of cerebral myxomatous aneurysms.

\section{CONFLICTS OF INTEREST}

No potential conflict of interest relevant to this article was reported.

\section{REFERENCES}

1. Aggarwal SK, Barik R, Sarma TC, Iyer VR, Sai V, Mishra J, et al.: Clinical presentation and investigation findings in cardiac myxomas: new insights from the developing world. Am Heart J 154:1102-1107, 2007

2. Ashalatha R, Moosa A, Gupta AK, Krishna Manohar SR, Sandhyamani S: Cerebral aneurysms in atrial myxoma: a delayed, rare manifestation. Neurol India 53:216-218, 2005

3. Branscheidt M, Frontzek K, Bozinov O, Valavanis A, Rushing EJ, Weller M, et al.: Etoposide/carboplatin chemotherapy for the treatment of metastatic myxomatous cerebral aneurysms. J Neurol 261:828-830, 2014

4. Burke A, Tavora F: The $2015 \mathrm{WHO}$ classification of tumors of the heart and pericardium. J Thorac Oncol 11:441-452, 2016

5. Dhawan SR, Gupta A, Gupta V, Singhi PD: Multiple intracranial aneurysms in HIV infection. Indian J Pediatr 83:852-854, 2016

6. Ekinci EI, Donnan GA: Neurological manifestations of cardiac myxoma: a review of the literature and report of cases. Intern Med J 34:243-249, 2004

7. Flores PL, Haglund F, Bhogal P, Yeo Leong Litt L, Södermann M: The dynamic natural history of cerebral aneurysms from cardiac myxomas: A review of the natural history of myxomatous aneurysms. Interv Neuroradiol 24:277-283, 2018

8. Herbst M, Wattjes MP, Urbach H, Inhetvin-Hutter C, Becker D, Klockgether T, et al.: Cerebral embolism from left atrial myxoma leading to cerebral and retinal aneurysms: a case report. AJNR Am J Neuroradiol 26:666-669, 2005

9. Josephson SA, Johnston SC: Multiple stable fusiform intracranial aneurysms following atrial myxoma. Neurology 64:526, 2005

10. Lee VH, Connolly HM, Brown RD, Jr.: Central nervous system manifestations of cardiac myxoma. Arch Neurol 64:1115-1120, 2007

11. Radoi MP, Stefanescu F, Arsene D: Brain metastases and multiple cerebral aneurysms from cardiac myxoma: case report and review of the literature. Br J Neurosurg 26:893-895, 2012

12. Sabolek M, Bachus-Banaschak K, Bachus R, Arnold G, Storch A: Multiple cerebral aneurysms as delayed complication of left cardiac myxoma: a case report and review. Acta Neurol Scand 111:345-350, 2005

13. Sedat J, Chau Y, Dunac A, Gomez N, Suissa L, Mahagne MH: Multiple cerebral aneurysms caused by cardiac myxoma. A case report and present state of knowledge. Interv Neuroradiol 13: 179-184, 2007

14. Stock K: Multiple cerebral aneurysms in a patient with recurrent cardiac myxomas. A case report. Interv Neuroradiol 10:335340, 2004

15. Tamulevičiūtė E, Taeshineetanakul P, Terbrugge K, Krings T: Myxomatous aneurysms: a case report and literature review. Interv Neuroradiol 17:188-194, 2011

16. Thawani JP, Nayak NR, Pisapia JM, Petrov D, Pukenas BA, Hurst RW, et al.: Aneurysmal vasculopathy in human-acquired immunodeficiency virus-infected adults: Imaging case series and review of the literature. Interv Neuroradiol 21:441-450, 2015 